\title{
Detección de metales pesados en bovinos, en los valles de los rios Sinú y San Jorge, departamento de Córdoba, Colombia
}

\section{Detection of heavy metals in cattle, in the valleys of the Sinu and San Jorge rivers, department of Cordoba, Colombia}

\author{
Aura Madero G, ${ }^{1 *}$ M.Sc, José Marrugo N, ${ }^{1}$ Ph.D.
}

${ }^{1}$ Sistema Estatal de Universidades del Caribe, SUE CARIBE. Maestría en Ciencias Ambientales. Grupo de aguas, Química Aplicada y Ambiental, Universidad de Córdoba, Montería, Colombia. *Correspondencia: auramadero@hotmail.com

Recibido: Agosto de 2009; Aceptado: Julio de 2010.

\section{RESUMEN}

Objetivo. Evaluar la presencia de metales pesados: plomo(Pb), cobre (Cu), cadmio (Cd) y mercurio $(\mathrm{Hg})$ en hígado y músculo pectoral derecho de bovino, procedentes de fincas de la zona del Sinú y San Jorge. Materiales y métodos. Se muestrearon bovinos machos, adultos, de raza cebú mestizo, entre 2 a 7 años los cuales fueron sacrificados para consumo humano durante seis meses. Las muestras se analizaron mediante: espectroscopia de absorción atómica, empleando lámparas de cátodo hueco y un quemador de aire acetileno para determinar $\mathrm{Cu}$; vapor frío para la detección de $\mathrm{Hg}$ y polarografía para la detección de $\mathrm{Cd}$ y $\mathrm{Pb}$. Resultados. Se observó que Los rangos de $\mathrm{Hg}, \mathrm{Cu}, \mathrm{Cd}$ y $\mathrm{Pb}$ estaban por debajo de los parámetros de referencia de la Comisión Europea y la Norma Oficial Mexicana. En el $4 \%$ de las muestras se encontraron niveles de $\mathrm{Cu}$ no permisibles para consumo humano. Conclusiones. La industria cárnica de la región presenta una gran potencialidad para acceder a mercados internacionales, debido a que el $96 \%$ de las reses presentaron niveles de metales por debajo de los límites permisibles de México y Europa. A pesar de que los niveles de mercurio se encuentran en los límites permisibles, la evaluación del riesgo basado en el índice de peligrosidad, sugiere que el consumo diario de 100 gramos de productos cárnicos con niveles de 0.08 ppm, podría incrementar el riesgo de envenenamiento a una persona de $70 \mathrm{Kg}$.

Palabras clave: Metales pesados, bovinos, seguridad alimentaria. (Fuentes: MeSH, AIMS) 


\section{ABSTRACT}

Objective. To evaluate the presence of heavy metals: lead $(\mathrm{Pb})$, copper $(\mathrm{Cu})$, cadmium $(\mathrm{Cd})$, and mercury $(\mathrm{Hg})$ in the liver and right pectoral muscle veal, from farms located in the Sinu and San Jorge area. Materials and methods. Adults, crossbred zebu, male cattle, approximated 2 to 7 years of age were sampled. SAMPLES were taken during a period of six months from animals slaughtered for human consumption. The metals were assessed by atomic absorption spectroscopy, using lights and tasted a hollow air-acetylene burner to determine $\mathrm{Cu}$; cold vapor for the detection of $\mathrm{Hg}$ and polarography to detect $\mathrm{Cd}$ and $\mathrm{Pb}$. Results. The ranges of $\mathrm{Hg}, \mathrm{Cu}, \mathrm{Pb}$ and $\mathrm{Cd}$ are below the benchmarks of the European Commission and the Norma Oficial Mexicana. Levels were not permissible for human consumption of $\mathrm{Cu}$ in $4 \%$ of the samples. Conclusions. The meat industry in the region has great potential to access international markets, since $96 \%$ of cattle had levels of metals below the permissible limits of Mexico and Europe. While $\mathrm{Hg}$ levels are in the permissible limits, risk assessment based on the hazard index suggested that the daily consumption of 100 grams of meat products with levels of $0.08 \mathrm{ppm}$, might increase the risk of poisoning for a person of $70 \mathrm{~kg}$ weight.

Key words: Heavy metals, cattle, food safety. (Sources: MeSH, AIMS)

\section{INTRODUCCIÓN}

En Colombia, el departamento de Córdoba se destaca por las actividades agrícolas y ganaderas. El $70 \%$ del territorio del departamento, está destinado a actividades de ganadería extensiva (1). Según la Encuesta Nacional Agropecuaria - ENA; para el año 2007 el inventario total de ganado bovino en el departamento de Córdoba es de 2.366.960 reses, distribuidas en una población de 1.526.899 machos y 840.061 hembras (2). Esta población se encuentra localizada en un $95 \%$, en las zonas de la cuenca del río Sinú y la cuenca del río San Jorge, el restante $5 \%$ se encuentra ubicada en la cuenca de Canalete y Costanera, según el ordenamiento ambiental departamental realizado por la Corporación Autónoma regional de los valles del Sinú y del San Jorge (CVS) $(3,4)$.

A nivel nacional e internacional se han creado una serie de normas con el fin de que se garantice a través de la cadena de producción, la inocuidad de los productos cárnicos y lácteos. Dentro de esta normatividad cobra un papel destacado el control de los metales pesados en especies bovinas con el fin de mitigar el impacto ambiental e incentivar la producción más limpia, las buenas prácticas ganaderas y la llamada "agricultura orgánica" o "agricultura limpia" $(5,6)$. Frente al pobre desarrollo de estas alternativas de producción, hay que destacar el apoyo que puede brindar la implementación de estudios científicos en éste campo, entre ellos la detección de metales.

Los metales pesados son especies químicas no degradables. Por tal motivo, una vez arrojados al medio ambiente, sólo pueden distribuirse entre los entornos aire - agua - suelo, a veces cambiando su estado de oxidación, o incorporarse a los seres vivos (7). La presencia de metales pesados en alimentos constituye un tema de actualidad debido a la contaminación de la cadena trófica involucrada y a los daños que ocasionan a la salud pública $(8,9)$.

Estudios de metales que se han realizado en el departamento de Córdoba, relacionados con la detección de niveles de mercurio en especies ícticas de la cuenca del río San Jorge, demuestran que en su mayoría, este metal sobrepasa el umbral estipulado (200 $\mathrm{ng} / \mathrm{g}$ ) para las poblaciones en riesgo establecido por la Organización Mundial de la Salud (OMS) (10), otros estudios evidencian la contaminación con mercurio en plantas, peces y sedimentos en algunos cuerpos de agua en la región de 
la Mojana $(11,12)$. En el mismo sentido, se encontraron concentraciones apreciables de mercurio en aguas, plantas, peces y sedimentos de la Ciénaga de Ayapel (13). Las fuentes sugieren que toda la problemática de la contaminación con metales está asociada con las actividades mineras desarrolladas en la principal zona aurífera de Colombia y en la cuenca del Río San Jorge.

El objetivo del presente estudio fue evaluar la contaminación por metales en ganado bovino del Sinú y el San Jorge y establecer el riesgo potencial para la salud humana.

\section{MATERIALES Y MÉTODOS}

\section{Selección, recolección y tratamiento} de muestras. Para evitar falsos positivos debido a deficiencias en el sistemas de sacrificio, en este estudio, todas las muestras se obtuvieron de reses que llegaron a un frigorífico Clase I, que cumple con los requisitos legales y las disposiciones de los gremios y sectores dedicados a la ganadería en Colombia. 90 muestras se recolectaron durante un periodo de seis meses, entre septiembre del 2008 y marzo del 2009, y como requisito de inclusión de las especies bovinas se tuvo en cuenta:

- Que sean reses con las siguientes características; bovinos adultos, edad promedio 2-7 años, sexo macho, raza cebú mestiza siendo la más común Brahman mestizo por Bos taurus.

- Cuyas procedencias sean de las cuencas del río Sinú y del río San Jorge.

- Como grupo control fueron seleccionadas 20 reses que provenían de fincas inscritas en programa de buenas prácticas ganaderas (BPG) del Instituto Colombiano Agropecuario (ICA), este grupo se denominó: BPG de las cuales se tomaron 20 muestras procedentes de 4 fincas.

Para la selección de número de reses a analizar se empleo el método de muestreo aleatorio estratificado (14), seleccionando dos categorías (Sinú y San Jorge), a las cuales se le asignó una proporción igual teniendo en cuenta que en estas zonas se concentra el $95 \%$ del ganado de todo el departamento y la proporción de ganado bovino macho, tanto para la zona del Sinú como la del San Jorge es del 95\%. De este modo, e incluyendo el grupo control, se colectaron 90 muestras de musculo pectoral derecho y 90 muestras de hígado, en lotes de cinco reses de 18 fincas (Tabla 1 ).

Tabla 1. Descripción de las zonas de muestreo.

\begin{tabular}{cccc}
\hline GRUPOS & Códigos de fincas & $\mathbf{N}^{\circ}$ reses/finca & Municipio \\
\hline \multirow{6}{*}{ Sinú } & 6 & 5 & Tierralta \\
& 7 & 5 & San Pelayo \\
& 11 & 5 & Lorica \\
& 12 & 5 & Cereté \\
& 13 & 5 & Cereté \\
& 17 & 5 & Montería \\
& 18 & 5 & Montería \\
\hline \multirow{4}{*}{ San Jorge } & 1 & 5 & Ayapel \\
& 3 & 5 & Ayapel \\
& 4 & 5 & Buenavista \\
& 5 & 5 & Buenavista \\
& 9 & 5 & Montelibano \\
& 10 & 5 & Pueblo Nuevo \\
BPG & 2 & 5 & Ayapel \\
\hline \multirow{6}{*}{ Total } & 14 & 5 & Puerto Escondido \\
& 15 & 5 & Montería \\
& 16 & 5 & Montería \\
\hline
\end{tabular}

En total, se tomaron 180 muestras de 10 a $50 \mathrm{~g}$, las cuales fueron empacadas en bolsas plásticas etiquetadas y congeladas hasta su análisis, para la detección de mercurio $(\mathrm{Hg})$, cobre $(\mathrm{Cu})$, cadmio $(\mathrm{Cd})$ y plomo $(\mathrm{Pb})$.

Geoposición de fincas. De cada res muestreada se registró el número de lote y la fecha de muestreo, nombre de la finca y propietario, con esa información se obtuvo la geoposición en latitud y longitud.

Estos datos fueron transformados mediante el software Geocal y se exportaron a dbf y al Sofware Arc gis 9.2, de esta manera se corroboró la localización dentro del departamento de Córdoba y se construyó un mapa con la ubicación geográfica de las fincas muestreadas, mostrando la relación de estos puntos con las zonas de alto, medio y bajo riesgo de inundación según los registros publicados por la CVS $(3,4)$. 
Dado que es importante establecer si existe alguna relación entre Las zonas inundables, según la ubicación geográfica se clasificaron las fincas en inundable y no inundable. Esto parámetros se evaluaron debido a que en la inundación se producen condiciones de anoxia, las cuales conducen a la formación de ácido de sulfuro volátil (AVS) y la unión de metales que se encuentran en el terreno. Este efecto produce variaciones espaciales temporales en los sedimentos de los ríos y las llanuras de inundación (15).

Detección de mercurio. Se tomaron para el análisis $0.5 \mathrm{~g}$ de muestra tanto de musculo como de hígado, las cuales fueron digeridas en una mescla oxidante de ácido sulfúrico-acido nítrico, a temperatura entre 90 y $100^{\circ} \mathrm{C}$ por un tiempo máximo de tres horas. Se dejó enfriar, y se adicionó una solución saturada de $\mathrm{KMnO}_{4}$, seguidamente se calentó a la misma temperatura por media hora, se enfrió y se adicionó una solución de cloruro de hidroxilamina al $1.5 \%$ para neutralizar el exceso de agente oxidante. A la solución con la muestra digerida, se agregó cloruro de estaño $\left(\mathrm{SnCl}_{2}\right)$ al $10 \%$ para reducir el $\mathrm{Hg}$ en su totalidad a su forma elemental, luego se empleó espectroscopia de absorción atómica mediante la técnica de vapor frío adaptada de Sadiq (16) y Environmental Protection Agency (EPA) (17).

Detección de cobre. Se cuantificó por el método de absorción atómica con llama adaptando una digestión de muestra acorde a Hülya (18), previamente validado en el laboratorio con el siguiente procedimiento de digestión y análisis: Se pesaron $0.5 \mathrm{~g}$ de muestra de tejido en un erlenmeyer de reacción previamente lavados con una solución 1:1 de $\mathrm{HNO}_{3}$, enjuagados con agua desionizada y totalmente secos. Se adicionaron $8.0 \mathrm{ml}$ de una mezcla ácida 6:2 de $\mathrm{HNO}_{3}-\mathrm{HCl}$ y se calentó la mescla en un baño de agua termostatado a $95^{\circ} \mathrm{C}$ por 3 horas. Luego de enfriarse, la solución se filtró por una membrana de $0.45 \mu \mathrm{m}$, se aforó hasta $25.0 \mathrm{~mL}$ con agua acidulada ( $0.15 \%$ de ácido nítrico) y se procedió a su análisis. Se registró el valor de máxima absorbancia de la muestra en el equipo de absorción atómica (Thermo Electron Corporation, S4-SERIE con flujo de aireacetileno y corrector de fondo de Deuterio) y se determinó su concentración de $\mathrm{Cu}$ utilizando la curva de calibración. Los análisis fueron realizados por duplicado y los resultados se expresaron en $\mu \mathrm{g} / \mathrm{g}$.

Detección de cadmio y plomo. Estos metales se cuantificaron por el método de voltametría de redisolución anódica de pulso diferencial adaptando una digestión de muestra acorde a Hülya $(18,19)$, previamente validado en el laboratorio con el siguiente procedimiento de digestión y análisis: Se pesaron $0.5 \mathrm{~g}$ de muestra de tejido en un erlenmeyer de reacción previamente lavados con una solución 1:1 de $\mathrm{HNO} 3$, enjuagados con agua desionizada y totalmente secos. Se adicionaron $8.0 \mathrm{ml}$ de una mezcla ácida 6:2 de $\mathrm{HNO} 3-\mathrm{HCl}$ y se calentó la mescla en un baño de agua termostatado a $95^{\circ} \mathrm{C}$ por 3 horas. Luego de enfriarse, la solución se filtró por una membrana de $0.45 \mu \mathrm{m}$, se aforó hasta 25.0 $\mathrm{ml}$ con agua acidulada $(0.15 \%$ de ácido nítrico), se depositaron $10.0 \mathrm{ml}$ de esta muestra diluida en la celda electroquímica y se adicionaron $2 \mathrm{ml}$ de solución buffer de acetato de sodio-cloruro de sodio. Se registró el valor de intensidad de la muestra en el equipo polarógrafo (Polarógrafo Metrhom Procesador 747 con V Stand 746) y determinó su concentración de Cd y $\mathrm{Pb}$ utilizando la curva de calibración. Los análisis se realizaron por duplicado y los resultados se expresaron en $\mu \mathrm{g} / \mathrm{g}$.

Criterios de control de calidad. Se tuvieron en cuenta: linealidad, exactitud, precisión, límite de detección y porcentaje de recuperación. Los límites de detección de los metales fueron: $0.005 \mu \mathrm{g} / \mathrm{g}$ para $\mathrm{Cd} \mathrm{y} \mathrm{Pb}, 2.0 \mu \mathrm{g} / \mathrm{g}$ para $\mathrm{Cu}$ y $0.01 \mu \mathrm{g} / \mathrm{g}$ para $\mathrm{Hg}$. Simultáneamente se proceso un estándar de tejido IAEA-407 (International Atomic Energy Agency), para determinar el porcentaje de extracción de las muestras y el coeficiente de variación (Tabla 2).

Índice de peligrosidad (HI). El HI se definió como la relación entre el nivel de exposición de una sustancia en particular (E) y una dosis de referencia (RfD) ó 
Tabla 2. Análisis del material de referencia (IAEA-407) para comprobación de los métodos analíticos.

\begin{tabular}{cccccc}
\cline { 2 - 5 } Analito & $\begin{array}{c}\text { Valor } \\
\text { referencia } \\
(\boldsymbol{\mu} / \mathbf{g})\end{array}$ & $\begin{array}{c}\text { Valor } \\
\text { encontrado } \\
(\boldsymbol{\mu} / \mathbf{g})\end{array}$ & CV (\%) & $\%$ Método \\
\hline $\mathrm{Cd}$ & $0.043 \pm 0.008$ & $0.040 \pm 0.009$ & 2.2 & 97.3 & ASV \\
$\mathrm{Pb}$ & $0.065 \pm 0.007$ & $0.070 \pm 0.006$ & 1.8 & 98.7 & ASV \\
$\mathrm{Cu}$ & $2.34 \pm 0.16$ & $2.46 \pm 0.13$ & 2.4 & 96.4 & A.A \\
$\mathrm{Hg}$ & $4.64 \pm 0.26$ & $4.60 \pm 0.21$ & 1.1 & 98.8 & CVAS \\
\hline
\end{tabular}

\%CV: Coeficiente de Variación, \%R: Porcentaje de recuperación, A.A : Absorción atómica, CVAS: espectrofotometría de absorción atómica de vapor frio, ASV: Voltametría de redisolución anódica (Método polarográfico).

dosis de riesgo mínimo (MRL). El E para el consumo de $\mathrm{MeHg}$ se calculó utilizando la fórmula: $\mathrm{E}=\mathrm{CXI} / \mathrm{W}$, donde $\mathrm{C}$ es la concentración promedio de $\mathrm{MeHg}$ de la especie en consideración. Considerando que el $90 \%$ del $\mathrm{Hg}-\mathrm{T}$ encontrado en las muestras es $\mathrm{MeHg}$, entonces $\mathrm{C}=0.90 \times \mathrm{Hg}-\mathrm{T}$ (20); I es la ingesta diaria de carne ó hígado establecida en $100 \mathrm{~g} /$ día; y $\mathrm{W}$ es el peso promedio de una persona adulta normal $(70 \mathrm{~kg})$. La EPA (Environmental Protection Agency) ha propuesto una RfD para $\mathrm{MeHg}$ de $0.0001 \mathrm{mg} / \mathrm{kg} /$ día (21), para el Cd $0.001 \mathrm{mg} / \mathrm{kg} /$ día (22). En el caso del Pb y $\mathrm{Cu}$, no existen valores de RfD establecidos por la EPA, por lo que se tuvo en cuenta los reportes encontrados en la literatura; de este modo, la RfD para $\mathrm{Pb}$ es 0.000357 $\mathrm{mg} / \mathrm{kg} /$ día (23) y para Cu es $0.01 \mathrm{mg} / \mathrm{kg} /$ día (24).

Análisis estadístico. Se realizó un ANOVA bajo un modelo de efectos anidados y prueba least square mean de comparación de medias, para evaluar si existía diferencia entre las zonas y los tipos de terreno. Los datos fueron analizados para comprobar su distribución normal (prueba Shapiro-Wilk) y homogeneidad de varianzas (prueba de Levene). Todos los análisis estadísticos se desarrollaron en el programa SAS (Statistical Analisys System) versión 9.0 y para todos los análisis el criterio de significancia estadística se definió como $p<0.05$. Los resultados de expresaron en $\mu \mathrm{g} / \mathrm{g}$ de peso.

\section{RESULTADOS}

Con los datos obtenidos de la posición geográfica de las fincas muestreadas, se elaboró un mapa de su ubicación en el departamento y su relación con las zonas en riesgo de inundación (Figura 1).

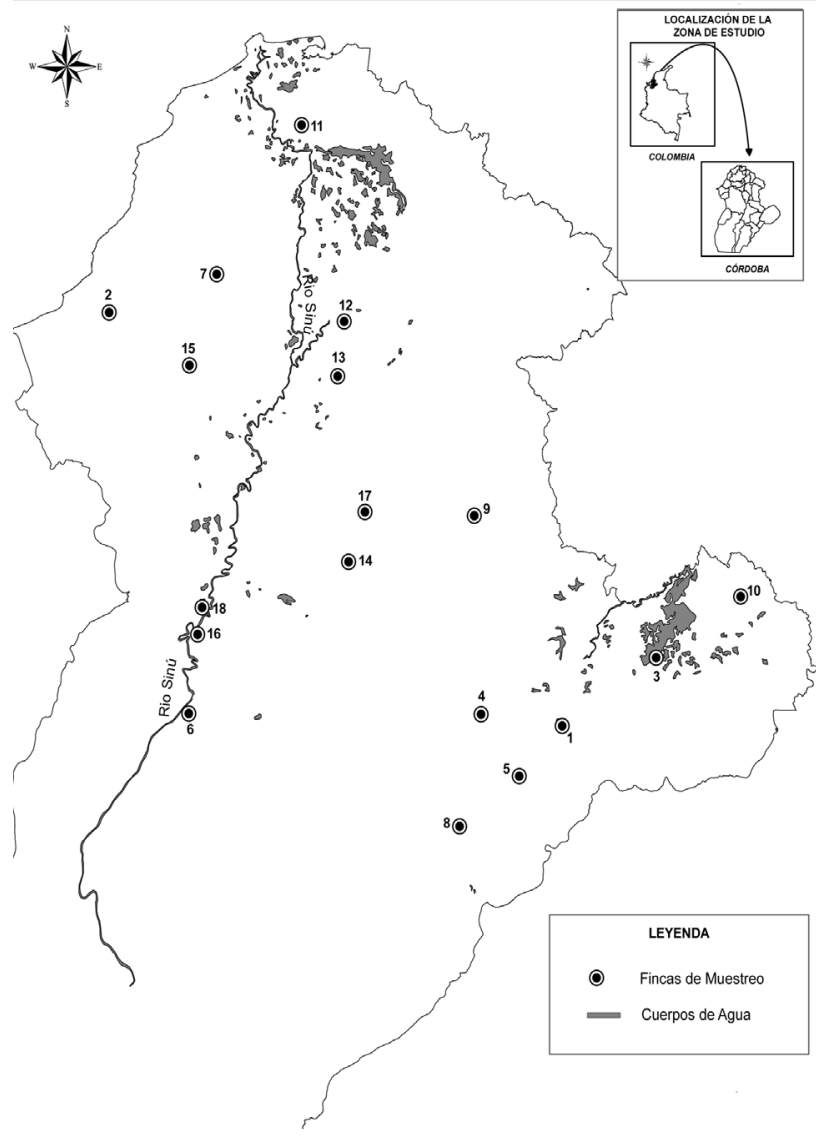

Figura 1. Localización geográfica de las fincas muestreadas.

Las concentraciones medias de $\mathrm{Hg}, \mathrm{Cd}, \mathrm{Pb}$ y $\mathrm{Cu}$ para el total de muestras tanto en musculo dorsal como en hígado de bovinos machos, fueron inferiores a los valores de referencia de la Norma Mexicana (25) y la Comisión Europea-CE (26), excepto en dos muestras de musculo (2.2\%) y dos de hígado $(2.2 \%)$, que excedieron estos valores para la concentración de Cu (Tabla 3).

En cuanto a las diferentes zonas muestreadas y tipos de terreno, las mayores concentraciones de $\mathrm{Hg}$ tanto en musculo como en hígado se registraron en el San Jorge y las BPG sin diferencias estadísticas 
Tabla 3. Concentraciones medias y desviación estándar $(M \pm S D)$ de metales en musculo dorsal e hígado de bovinos machos, para el total de muestras.

\begin{tabular}{|c|c|c|c|c|c|c|c|}
\hline \multirow[t]{2}{*}{ Metal } & \multirow[t]{2}{*}{ Tejido } & \multicolumn{2}{|c|}{$\begin{array}{c}\text { Concentración de } \\
\text { metales }(\mu / g)\end{array}$} & \multirow[t]{2}{*}{$N M^{a}$} & \multirow[t]{2}{*}{$\mathrm{CE}^{\mathrm{b}}$} & \multirow[t]{2}{*}{$N M>L^{c}$} & \multirow[t]{2}{*}{ c $n$} \\
\hline & & $\mathrm{M} \pm \mathrm{SD}$ & Intervalo & & & & \\
\hline \multirow{2}{*}{$\mathrm{Hg}$} & Musculo & $0.027 \pm 0.019$ & $0.008-0.104$ & 0.7 & ND & 0 & 90 \\
\hline & Hígado & $0.028 \pm 0.025$ & $0.003-0.110$ & 1.4 & ND & 0 & 90 \\
\hline \multirow{2}{*}{$\mathrm{Cd}$} & Musculo & $0.007 \pm 0.004$ & $0.001-0.016$ & 2 & 0.05 & 0 & 90 \\
\hline & Hígado & $0.011 \pm 0.006$ & $0.001-0.026$ & 10 & 0.5 & 0 & 90 \\
\hline \multirow{2}{*}{$\mathrm{Pb}$} & Musculo & $0.044 \pm 0.022$ & $0.007-0.095$ & 0.5 & 0.1 & 0 & 90 \\
\hline & Hígado & $0.059 \pm 0.020$ & $0.020-0.098$ & 2 & 0.5 & 0 & 90 \\
\hline \multirow{2}{*}{$\mathrm{Cu}$} & Musculo & $0.155 \pm 0.499$ & $0.000-2.800$ & 2 & ND & 2 & 90 \\
\hline & Hígado & $44.23 \pm 24.32$ & $0.17-177.30$ & 60 & ND & 2 & 90 \\
\hline
\end{tabular}

${ }^{a}=$ Limite permisible por la Norma Oficial Mexicana (norma 004-ZOO-1994). b=Límite permisible por la Comisión Europea $\left(\mathrm{N}^{\circ} 466 / 2001\right)$. ${ }^{\mathrm{c}}=$ Número de muestras que excedieron el limite permisible por las normas descritas arriba

significativas entre ellas $(p>0.05)$, pero estadísticamente diferentes a la zona del Sinú $(p<0.05)$. La mayor concentración de $\mathrm{Cu}$ en hígado se registró en el San Jorge con diferencias significativas respecto al Sinú y las BPG $(<0.05)$; no se encontraron diferencias estadísticas significativas en las concentraciones de Cu en musculo, ni de Cd y $\mathrm{Pb}$ en musculo e hígado entre las diferentes zonas de muestreo (Tabla 4). Igualmente no se registraron diferencias estadísticas significativas en las concentraciones de $\mathrm{Hg}$,
$\mathrm{Cu}, \mathrm{Cd} \mathrm{y} \mathrm{Pb}$ en musculo e hígado entre los tipos de terrenos (Tabla 4). En todos los casos, las concentraciones de todos los metales evaluados fueron inferiores a los valores de referencia de la norma mexica y de la CE, excepto para las concentraciones de $\mathrm{Cu}$ en musculo que estuvieron en el límite de la norma, tanto en las diferentes zonas y tipos de terreno.

Se registraron correlaciones positivas significativas entre las concentraciones de $\mathrm{Cd} \mathrm{y} \mathrm{Hg}$ en musculo e hígado, en las diferentes zonas y tipos de terreno, excepto para las concentraciones de $\mathrm{Hg}$ en la zona del Sinú (Tabla 5). No se registraron correlaciones significativas entre las concentraciones de $\mathrm{Cu}$ y $\mathrm{Pb}$ en musculo e hígado en las diferentes zonas y tipos de terreno, excepto para las concentraciones de $\mathrm{Pb}$ en el terreno inundable (Tabla 5 ).

Tabla 5. Coeficiente de correlación lineal para el contenido de metales entre músculo e hígado.

\begin{tabular}{|c|c|c|c|c|c|c|}
\hline \multirow[b]{2}{*}{ Metal } & \multicolumn{3}{|c|}{ Zona } & \multicolumn{2}{|c|}{ Tipo terreno } & \multirow[b]{2}{*}{ General } \\
\hline & Sinú & San Jorge & BPG & Inundable & $\begin{array}{c}\text { No } \\
\text { inundable }\end{array}$ & \\
\hline $\mathrm{Hg}$ & $0.17 \mathrm{~ns}$ & $0.89 * * *$ & $0.83 * * *$ & $0.88 * * *$ & $0.89 * * *$ & $0.88 * * *$ \\
\hline $\mathrm{Cu}$ & & $0.01 \mathrm{~ns}$ & & $0.04 \mathrm{~ns}$ & & $0.03 \mathrm{~ns}$ \\
\hline $\mathrm{Cd}$ & $0.99 * * *$ & $0.97 * * *$ & $0.99 * * *$ & $0.99 * * *$ & $0.98 * * *$ & $0.99 * * *$ \\
\hline $\mathrm{Pb}$ & $0.24 \mathrm{~ns}$ & $0.27 \mathrm{~ns}$ & $0.16 \mathrm{~ns}$ & $0.42 *$ & 0.14 ns & $0.25 *$ \\
\hline
\end{tabular}

$n s=$ No significativa $(p>0.05), *=$ Significativa $(p<0.05)$, $* * *=$ Altamente significativa $(\mathrm{p}<0.01)$.

Tabla 4. Comparación de las concentraciones de metales en musculo dorsal (M) e hígado (H) de bovinos machos, entre zonas de muestreo (Sinú y San Jorge) y entre tipos de terrenos (inundables y no inundables). Letras diferentes entre columnas muestran diferencias estadísticas significativas $(p<0.05)$.

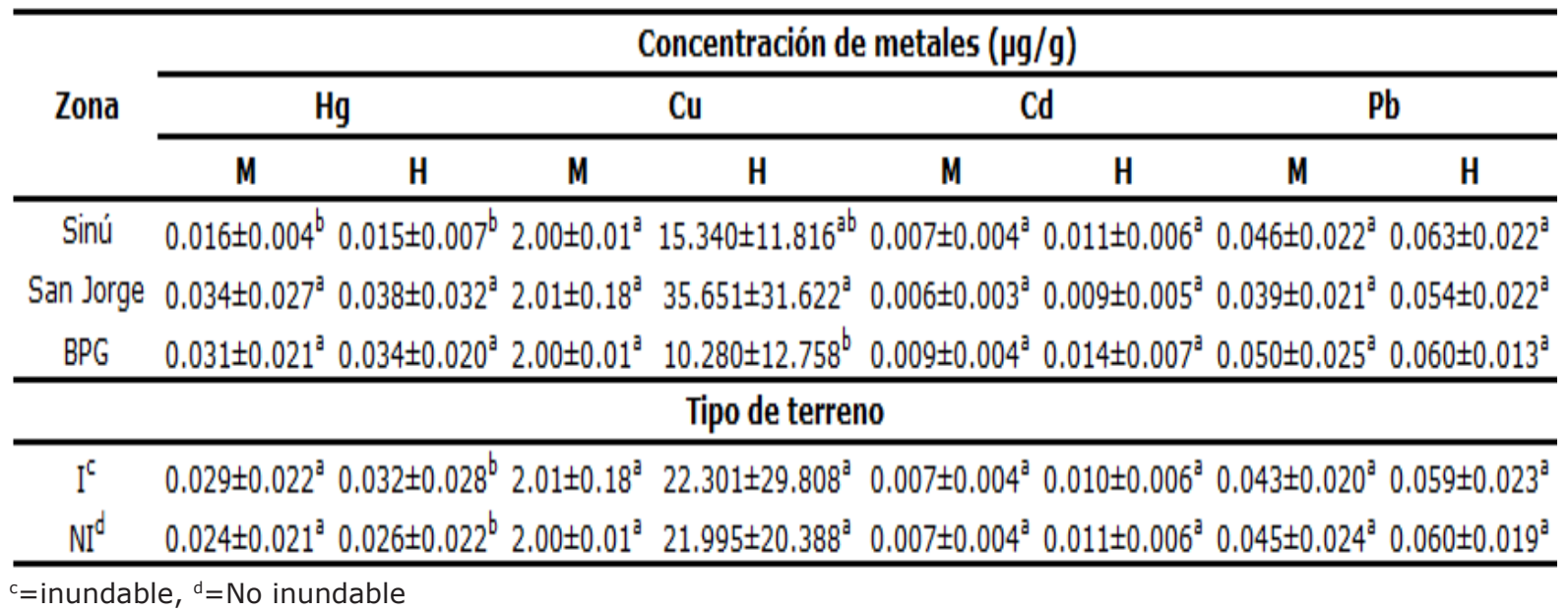


La tabla 6, presenta el análisis de correlación lineal entre los contenidos de metales en todas las muestras en forma independiente para musculo e hígado. Los resultados muestran una positiva significativa entre las concentraciones de $\mathrm{Cd}$ y $\mathrm{Pb}$ tanto en musculo como en hígado. Los demás metales no se correlacionaron entre sí.

Tabla 6. Coeficiente de correlación lineal para el contenido de metales en general.

\begin{tabular}{ccccccc}
\hline & \multicolumn{3}{c}{ Musculo } & \multicolumn{3}{c}{ Hígado } \\
\cline { 2 - 6 } Metal & $\mathbf{H g}$ & $\mathrm{Cu}$ & $\mathrm{Cd}$ & $\mathrm{Hg}$ & $\mathrm{Cu}$ & $\mathbf{C d}$ \\
\hline $\mathrm{Cu}$ & $-0.03 \mathrm{~ns}$ & \multicolumn{4}{c}{$-0.04 \mathrm{~ns}$} \\
$\mathrm{Cd}$ & $-0.12 \mathrm{~ns}$ & $0.12 \mathrm{~ns}$ & & $-0.10 \mathrm{~ns}$ & $-0.11 \mathrm{~ns}$ \\
$\mathrm{~Pb}$ & $-0.12 \mathrm{~ns}$ & $0.12 \mathrm{~ns}$ & $0.95^{* *}$ & $-0.18 \mathrm{~ns}$ & $-0.08 \mathrm{~ns}$ & 0.22 \\
\hline
\end{tabular}

ns $=$ No significativa $(p>0.05), *=$ Significativa $(p<0.05)$, $* *=$ Altamente significativa $(\mathrm{p}<0.01)$

El HI, excedió la unidad (valor crítico) para el consumo diario de $100 \mathrm{~g}$ tanto de musculo como de hígado con las concentraciones de $\mathrm{Hg}$ descritas en este estudio, y también para el consumo diario de $100 \mathrm{~g}$ de musculo con las concentraciones de $\mathrm{Cu}$ descritas en este estudio (Tabla 7).

Tabla 7. Valores de Índice de Peligrosidad (HI) estimados para un consumo diario de $100 \mathrm{~g}$ (musculo e hígado) para el total de muestras.

\begin{tabular}{ccccc}
\hline \multirow{2}{*}{ METAL } & \multicolumn{2}{c}{ HIGADO } & \multicolumn{2}{c}{ MUSCULO } \\
\cline { 2 - 5 } & $\begin{array}{c}\text { Intervalo } \\
\text { (min-max) }\end{array}$ & HI & $\begin{array}{r}\text { Intervalo } \\
\text { (min-max })\end{array}$ & HI \\
\hline \multirow{2}{*}{$\mathrm{Hg}$} & $0.008^{-}$ & $0.103-$ & $0.003-$ & $0.038^{-}$ \\
& 0.104 & 1.337 & 0.110 & 1.28 \\
$\mathrm{Cd}$ & $0.001-$ & $0.001-$ & $0.001-$ & $0.001-$ \\
& 0.016 & 0.023 & 0.026 & 0.037 \\
$\mathrm{Fb}$ & $0.007-$ & $0.028-$ & $0.020-$ & $0.080-$ \\
& 0.095 & 0.380 & 0.098 & 0.392 \\
$\mathrm{C} \mathrm{Cu}$ & $0.000-$ & $0.000-$ & $0.170-$ & $0.024-$ \\
& 2.800 & 0.400 & 177.3 & 25.33 \\
\hline
\end{tabular}

\section{DISCUSIÓN}

Las concentraciones hepáticas de metales pesados en ganado bovino también han sido evaluadas por otros investigadores. Para el $\mathrm{Pb},(27)$ registró $0.00-0.26 \mathrm{mg} / \mathrm{kg}$; (28), $15.30 \pm 1.14 \mathrm{mg} / \mathrm{kg}$ (29), no detectables (ND) $-411 \mu \mathrm{g} / \mathrm{kg}$ para zonas industriales y ND-174 $\mu \mathrm{g} / \mathrm{kg}$ para zonas rurales (como es el caso del presente estudio); (30), ND-509 $\mu \mathrm{g} / \mathrm{kg}$ (31), $0.09-7.32 \mathrm{mg} / \mathrm{kg}$. Las concentraciones hepáticas de $\mathrm{Pb}$ registradas en este estudio (0.020-0.098 $\mu \mathrm{g} / \mathrm{g})$, encajan dentro de los valores antes descritos, excepto con los de Liu (28), los cuales son marcadamente superiores.

Los niveles hepáticos de $\mathrm{Cd}$ reportados en los estudios citados, variaron entre $3.39-131 \mu \mathrm{g} / \mathrm{kg}$ para zonas industriales y $6.43-221 \mu \mathrm{g} / \mathrm{kg}$ para zonas rurales (29); $\mathrm{ND}-85.5 \mu \mathrm{g} / \mathrm{kg}(30) ; 0.03-0.13 \mathrm{mg} / \mathrm{kg}(27)$; $7.92 \pm 2.36 \mathrm{mg} / \mathrm{kg}$ (28); $0.001-0.02 \mathrm{mg} / \mathrm{kg}$ (31). Estas concentraciones son similares a la reportadas en este estudio (0.001-0.026 $\mu \mathrm{g} \mathrm{Cd} / \mathrm{g})$, excepto por los mayores valores de Liu (28).

Los niveles hepáticos de $\mathrm{Cu}$ reportados por estos mismo autores variaron entre $1.86-137 \mathrm{mg} / \mathrm{kg}$ para zonas industriales y 2.28-139 mg/kg para zonas rurales (29); $3.72-38 \mathrm{mg} / \mathrm{kg}$ (30); 0.46-4.89 mg/kg (27); $284.1 \pm 35.9 \mu \mathrm{g} / \mathrm{kg}$ (28); 0.03-2.20 $\mathrm{mg} / \mathrm{kg}$ (31). Las concentraciones de $\mathrm{Cu}$ $(0.17-177.30 \mu \mathrm{g} / \mathrm{g})$ reportadas en este estudio en hígado de bovinos, están igualmente en acuerdo con los reportes descritos, destacando las diferencias con el valor registrado por Liu (28).

La tabla 8 presenta los valores hepáticos considerados tóxicos, para $\mathrm{Cd}, \mathrm{Pb}$ y $\mathrm{Cu}$. En general, las concentraciones hepáticas de estos metales registradas en el presente estudio, fueron inferiores a estos valores,

Tabla 8. Concentraciones de metales consideradas tóxicas en hígado de ganado. Datos tomados de la revisión de Reis et al (32).

\begin{tabular}{ccc}
\hline Metal & Concentración (ppm) & Referencia \\
\hline $\mathrm{Cd}$ & $50-160$ & Suttle, 2001 \\
$\mathrm{Pb}$ & oct-20 & Radostits, 2007 \\
$\mathrm{Cu}$ & 1905 maximo & Radostits, 2007 \\
\hline
\end{tabular}


lo cual estaría indicando que no hay efectos tóxicos de los mismos que afecten la salud del ganado en las zonas evaluadas. Sin embargo, se debe resaltar que la exposición crónica a estos elementos en concentraciones relativamente menores que los niveles tóxicos, pueden generar a largo plazo, algunas alteraciones en la condición de salud del ganado (32).

Las concentraciones de $\mathrm{Hg}$ tanto en musculo $(0.008-0.104 \mu \mathrm{g} / \mathrm{g})$ como en hígado $(0.003-0.110 \mu \mathrm{g} / \mathrm{g})$ registradas en el presente estudio, son superiores a las registradas por otros autores en musculo (ND - $4.69 \mu \mathrm{g} / \mathrm{kg}$ ) e hígado (ND-26.4 $\mu \mathrm{g} / \mathrm{kg}$ ) (33). Con relación a la alimentación bovina lo ideal es que no se detecte elementos tóxicos como el $\mathrm{Hg}$ y $\mathrm{Pb}$; sin embargo, a pesar de que el departamento de Córdoba no ha sido impactado fuertemente por la industrialización, se detectaron niveles de $\mathrm{Hg}$ y $\mathrm{Pb}$. Esta situación debe ser atendida con mayor atención para evitar el incremento de estos metales en el ganado y su potencial riesgo para la salud humana por consumo de productos cárnicos.

Respecto a las concentraciones de los elementos esenciales $\mathrm{Cu}$ y $\mathrm{Cd}$, algunos autores han señalado que síntomas de deficiencia por $\mathrm{Cu}$ apare $\neg$ cerían cuando los valores hepáticos llegan a ser menores o iguales a $20 \mathrm{ppm}$ y los síntomas por intoxicación se presentan cuando los valores son mayores de 50 ppm (34). En este estudio se encontró que en el $40 \%$ de las reses (36 reses) presentaron deficiencias en $\mathrm{Cu}$, las cuales se distribuyeron entre las diferentes zonas así: $27 \%$ en la zona del Sinú, $9 \%$ en la zona del San Jorge y $4 \%$ en fincas con BPG. Es decir, en la zona del San Jorge, se encuentra el mayor número de reses con concentraciones mayores de 20 y menores de 50 ppm, lo que sugiere que para algunas fincas de estas zonas no es necesaria la suplementación con $\mathrm{Cu}$. Se debe resaltar que altos niveles de $\mathrm{Cu}$ (177.3 y $113.1 \mathrm{ppm}$ ) en el hígado, registrados en fincas del San Jorge, fueron considerados atípicos y pueden deberse a casos puntuales y aislados de contaminación, los cuales no son representativos del los lotes muestreados por finca.
De otro lado, las fuertes correlaciones entre las concentraciones de $\mathrm{Pb}$ y $\mathrm{Cd}$ a nivel hepático y muscular, denotan una acción sinérgica entre estos dos metales, que puede ir desde un efecto acumulativo a nivel hepático sin presencia de manifestaciones clínicas en el animal (asintomático), hasta crisis agudas y muerte, con los respectivos riesgos en la salud pública que puede ocasionar (35). Afortunadamente en este estudio, las concentraciones de $\mathrm{Pb}$ y $\mathrm{Cd}$ fueron menores de 3 y 0.27 ppm respectivamente, lo que reflejan mínimos grados de contaminación para bo $\neg$ vinos clínicamente sanos criados en forma exten $\neg$ siva (36) y son como se expuso anteriormente, son inferiores a las concentraciones consideradas tóxica a nivel hepático (Tabla 8).

En cuanto a los efectos de la acumulación de los metales evaluados en la salud humana, el alto valor de HI (25.33) para el consumo de hígado con las concentraciones máximas de Cu registradas en este estudio, denotan posibles repercusiónes en la salud humana, aumentando el riesgo de sufrir de cáncer por bioacumulación de este metal. Las recomendaciones nutricionales para el consumo de $\mathrm{Cu}$ en humanos oscilan entre 1.5 y $3 \mathrm{mg} /$ día dependiendo del individuo. Así, haciendo una estimación del consumo de hígado de bovinos con concentraciones de $\mathrm{Cu}$ de 75 ppm (base seca), todo el requerimiento diario de $\mathrm{Cu}$ de una persona podría ser cubierto mediante la ingestión de sólo $40 \mathrm{~g}$ de este hígado (en base seca) (5). Desde este punto de vista, no sería aconsejable el consumo diario de hígado con las concentraciones máximas $\mathrm{Cu}$ registrada en este estudio. Sin embargo, es conveniente recordar que solo dos muestras excedieron los límites permisibles por la NOM (Tabla 3).

Aunque todas las muestras evaluadas mostraron niveles permisibles de mercurio, El HI estimado para el consumo tanto de musculo como de hígado con las concentraciones máximas de $\mathrm{Hg}$ registradas en este estudio, muestra un riesgo ligeramente incrementado para la salud de las personas (Tabla 7). Sin embargo, al igual que para el $\mathrm{Cu}$, se debe resaltar 
que esta situación solo se presenta para los máximos valores registrados (entre 0.008 y $0.11 \mu \mathrm{g} / \mathrm{g}$ que representan el $6 \%$ de las muestras), por tanto los valores promedios de concentración de $\mathrm{Hg}$ no representan riesgo para la salud huma.

En general, aunque en bajos niveles, es evidente la presencia de metales tóxicos como el $\mathrm{Pb}$ y $\mathrm{Hg}$ en ganado bovino de las zonas del Sinú y San Jorge; por tanto, para evitar que estos se incremente, y mantener controlados los niveles de los metales esenciales, se sugiere que las autoridades ambientales y de salud junto con el gremio de los ganaderos y el ICA, deberían evaluar el riesgo por metales al implementar las buenas prácticas ganaderas. Para ello es aconsejable iniciar con el diagnóstico del grado de contaminación por finca, luego establecer los puntos críticos de contaminación e implementar medidas preventivas.
La industria cárnica en el departamento de Córdoba, posee un gran potencial para acceder a los mercados de México y Europa, dado que el $96 \%$ de las reses presentaron niveles de metales por debajo de los límites permisibles de estos países; por tanto, en lo que respecta a los metales evaluados, la carne es apta para consumo humano.

Finalmente, con este estudio se evidenció el papel de las especies bovinas como biomonitor de la contaminación por metales en la cadena productiva, en donde un ambiente contaminado, unido a deficiencias en las buenas prácticas ganaderas con lleva a la alteración de los productos cárnicos los cuales repercuten en la salud humana.

\section{Agradecimientos}

Los autores agradecen a la empresa Frigosinú S.A, por el apoyo logístico para la realización del muestreo, y a la Universidad de Córdoba por el apoyo financiero del estudio.

\section{REFERENCIAS}

1. CVS. Corporación Autónoma regional de los valles del Sinú y San Jorge, Centro Nacional de Producción más limpia. Diagnóstico regional de producción más limpia. Montería, Colombia: CVS; 2006.

2. Ministerio de Agricultura y Desarrollo Rural. República de Colombia. Encuesta nacional Agropecuaria -ENA. [en línea] 2008. [Consultado mayo 20 del 2008]. URL disponible en: http:// www.agronet.gov.co/www/docs agronet/20095694411_ENA_2008.pdf.

3. CVS. Corporación Autónoma regional de los valles del Sinú y San Jorge. Diagnóstico Ambiental de la cuenca hidrográfica del río Sinú. Montería, Colombia: CVS; 2004.
4. CVS. Corporación Autónoma regional de los valles del Sinú y San Jorge. Diagnóstico Ambiental de la cuenca hidrográfica del río San Jorge. Montería, Colombia: CVS; 2005.

5. Alcocer V. M, Castellanos A, Herrera Francisco, Chel L. y Betancur D. Detección de metales pesados y dicloro difenil triclor etano en músculos y órganos de bovinos en Yucatan. Tec Pecu Mex 2007; 45(2): 237-247

6. Pulido JI. Guía para la implementación de las buenas prácticas ganaderas. Colombia: CORPOICA, Ministerio de Agricultura y Desarrollo; 2007. 
7. Vullo D. Microorganismos y metales pesados: una interacción en beneficio del medio ambiente. [Revista electrónica] Química Viva 2003; 2(3). [Consultado octubre 29 del 2008]. URL disponible en: http:// www.quimicaviva.qb.fcen.uba.ar/ Actualizaciones/metales/metales

8. Mubbasher Sabyr S, Waheed $\mathrm{K}$ and Hayat I. Effect of environmental pollution on quality of meat in district Bagh, Azad Kashmir. Pakistan Jourl of Nutr 2003; 2(2):98-101.

9. Rodríguez $H$, Sánchez $E$, Rodríguez M, Vidales J A., Acuña K A., Martínez Gavo y Rodríguez J. Metales pesados en leche cruda de bovino. México. [Revista electrónica] Salud Pública y Nutrición. 2005; 6(4). [Consultado octubre 15 del 2008]. URL disponible en: Http://www.respyn.uanl.mx/vi/4/ articulos/metales.

10. Marrugo J, Olivero J, Lans E, Benítez L. Total mercury and methylmercury concentrations in fish from the Mojana region of Colombia. Environ Geochem Health 2007; 3.

11. Olivero J, Jhonson B, Arguello E. Human exposure to mercury due to fish consumption in San Jorge river basin, Colombia (South America). Sci Total Environm 2002; 289:41- 47.

12. Olivero J, Johnson B, Mendoza C, Paz $\mathrm{R}$, Olivero R. Mercury in the aquatic environment of The Village of Caimito at The Mojana Region, North of Colombia. Water Air Soil Pollut 2004; 159:409-420.

13. Marrugo J. y Lans E. Impacto ambiental por contaminación con níquel, mercurio y cadmio en aguas, peces y sedimentos en la cuenca del río San Jorge, en el departamento de Córdoba. [Internet] 2006. [Consultado junio 21 del 2008]. URL disponible en: http:// academico.unicordoba.edu.co:8080/ dspace/ bitstream /123456789/407/1/ Informe+Final.pdf.
14. Portella J, Villeta, M. Muestreo Estadístico. Teoría y Ejemplos. Madrid: Ed CERSA; 2007; 125 -300.

15. Poot A, Gillissen F, Koelmans A.A. Effects of flow regime and flooding on heavy metal availability in sediment and soil of a dynamic river system. Environm Pollu 2007; 148:779-787.

16. Sadiq M, Zaidi T, Al-Mohana M. Sample weight and digestion temperature as critical factors in mercury determination in fish. Bull Environ Contam Toxicol 1991; 47:335-341.

17. EPA. Environmental protection Agency. Methods 245.1, 245.5 and 245.6 for determination of mercury. Cincinnati. Ohio (USA): EPA; 1994.

18. Hülya K. A and Erhan Ü. Heavy Metal Concentrations in Water, Sediment, Fish and Some Benthic Organisms from Tigris River, Turkey. Environ Monit Assess 2007; 131:323-337.

19. Skoog D.A, Holler F.J, West D.M y Crouch S.R. Métodos polarográficos y voltamétricos de impulsos. Fundamentos de Química Analítica. Octava Edición. Mexico: Thomson. 2005.

20. Marrugo J., Lans E., Benítez L. Hallazgo de mercurio en peces de la ciénaga de Ayapel, Córdoba, Colombia. Rev MVZ Córdoba 2007; 12(1):878-886.

21. EPA. Environmental protection Agency. CASRN 22967-92-6. Methylmercury. [Internet] 1997. [Consultado julio 22 del 2009]. URL disponible en: http://cfpub.epa.gov/ ncea/iris/ index. cfm? fuseaction=iris. showQuick View \& substance. $\mathrm{nmbr}=0073 \#$ reforal.

22. EPA. Environmental protection Agency. CASRN - 7440-43-9 cadmio. [Internet] 1994. [Consultado julio 22 del 2009]. URL disponible en: http:// www.epa.gov/iris/subst/0141.htm. 
23. Health Canada. Health based tolerable daily intakes/concentrations and tumorigenic doses/concentrations for priority substances. Canada, Ottawa: Minister of Supply and services; 1996.

24. ATSDR. Agency for Toxic Substances and Disease Registry. Minimal Risk Levels (MRLs) for Hazardous Substances. [Internet] 2008. [Consultado julio $22 \mathrm{del}$ 2009]. URL disponible en: http://www. atsdr.cdc.gov.

25. Norma Oficial Mexicana. NOM (Norma Oficial Mexicana) 004-ZOO-1994. Grasa, hígado, musculo y riñones en aves, bovinos, caprinos, cérvidos, equinos, ovinos y porcinos. Residuos tóxicos. Límite máximo permisible y procedimiento de muestreo. Mexico; Secretaría de Agricultura, Ganadería, Desarrollo Rural, Pesca y Alimentación; 1994.

26. Reglamento (CE) $1881 / 2006$ DE LA COMISIÓN de 19 de diciembre de 2006 por el que se fija el contenido máximo de determinados contaminantes en los productos alimenticios. Diario Oficial de la Unión Europea; 2006.

27. Iwegbue CMA. Heavy metal composition of livers and kidneys of cattle from Southern Nigeria. Vet Arch 2008; 78:401-410.

28. Liu ZP. Lead poisoning combined cadmium in sheep and horses in the vicinity of non-ferrous metal smelters. Sci Total Envirom 2003; 309:117-126.

29. Miranda M, Lopez-Alonso M, Castillo C, Hernandez J, Benedito JL. Effects of moderate pollution on toxic and trace metal level in calves from a polluted area of Norther Spain. Envirom Int 2005; 31:543-548.

30. Blanco-Penedo I, Cruz JM, LopezAlonso $M$, Miranda $M$, Castillo $C$ et al. Influence of copper status on the accumulation of toxic and essential metals in cattle. Envirom Int 2006; 32:901-906.
31. Nwude DO, Okoye PAC, Babayemi Jo. Assessment of heavy metal concentrations in the liver of cattle slaughter during three different seasons. Res J Envirom Sci 2011; 5(3):288-294.

32. Reis LSL, Pardo PE, Camargos AS, Oba E. Mineral element and heavy metal poisoning in animals. J Med Med Sci 2010; 1 (12):560-579.

33. Lopez-Alonso M, Benedito JL, Miranda M, Castillo C, Hernández J, Shore RF. Mercury concentration in cattle of NW Spain. Sci Total Envirom 2003; 302:93-100.

34. BOILA R.J, DEVLIN R.A, DRYSDALE L.E. The severity of hypocupremia in selected hers of beef cattle in north Western Manitoba. Can J Anim Sci 1984; 64:919-936.

35. Castillo, Mayela; Padilla, Adriana; Suniaga, José; Betancourt, Arquimedes y Marcano,Enid.. Análisis de la Lemna sp. Del lago de Maracaibo para su eventual utilización en la alimentación de rumiantes. Agricultura andina. 2005; 10:3-8. [Consultado 12 de Noviembre de 2009]. URL disponible en: http://ecotropicos.saber.ula.ve/ $\mathrm{db} /$ /ssaber/Edocs/pubelectronicas/ agricultura_andina/vol10/articulo1. pdf.

36. Palavicino $H$, Meléndez $R$, Bergqvist $A$, Gálvez L. Determinación de metales pesados $(\mathrm{Cu}, \mathrm{Mo}, \mathrm{Zn}, \mathrm{Cd}, \mathrm{Pb})$ en hígados de bovinos procedentes de la Región Metropolitana y VI,VII,VIII,IX,X y XI Regiones de Chile. [Revista Internet] 1991. Avances de Medicina Veterinaria. 1991; 6(1). [Consultado julio 22 del 2009]. URL disponible en: http://www. avancesveterinaria.uchile.cl/CDA /avan _vet_completa/0,1424,SCID\%253D9648 \%2526ISID\%253D472,00.html. 